\title{
Shell Structure and Shape Changes in Neutron Rich Krypton Isotopes
}

\author{
D. Mücher ${ }^{\mathrm{a}}$, J. Iwanicki ${ }^{\mathrm{b}}$, J. Jolie $^{\mathrm{a}}$, I. Stefanescu ${ }^{\mathrm{c}}$, J. Van de Walle $^{\mathrm{c}}$,
} F. Becker ${ }^{\mathrm{d}}$, U. Bergmann ${ }^{\mathrm{e}}$, A. Blazhev ${ }^{\mathrm{a}}$, E. Bouchez ${ }^{\mathrm{f}}$, P. Butler' ${ }^{\mathrm{g}}$, J. Cederkälle, T. Czosnyka b ${ }^{\text {, T. Davinson }}{ }^{\text {, }}$, J. Eberth ${ }^{\mathrm{a}}$, T. Faestermann ${ }^{\mathrm{i}}$, S. Franchoo ${ }^{e}$, C. Fransen a , J. Gerl ${ }^{d}$, R. Gernhäuser ${ }^{i}$, D. Habs ${ }^{j}$, R-D. Herzberg ${ }^{g}$, M. Huyse ${ }^{c}$, D. Jenkins ${ }^{\mathrm{k}}$, G. Jones ${ }^{\mathrm{g}}$, O. Kester ${ }^{\mathrm{j}}$, W. Korten ${ }^{\mathrm{f}}$, J. Kownacki ${ }^{\mathrm{b}}$, T. Krölli ${ }^{\mathrm{i}}$ R. Krücken ${ }^{\mathrm{i}}, \mathrm{Z}_{\text {. Liu }}{ }^{\mathrm{h}}, \mathrm{S}$. Mandal ${ }^{\mathrm{d}}$, P. Napiorkowski ${ }^{\mathrm{b}}$, T. Nilsson ${ }^{\mathrm{e}}$, N. Pietralla ${ }^{\mathrm{l}}$, G. Rainovski ${ }^{\mathrm{m}}$, H. Scheit ${ }^{\mathrm{n}}$,

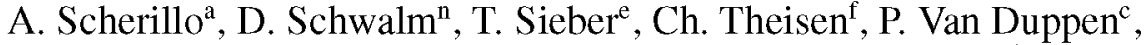
N. Warr ${ }^{\mathrm{a}}$, D. Weisshaar ${ }^{\mathrm{a}}$, F. Wenander ${ }^{\mathrm{e}}$, B. Wolf ${ }^{\mathrm{e}}$, P. Woods ${ }^{\mathrm{h}}$ and M. Zielinska ${ }^{b}$

\author{
${ }^{a}$ Institut für Kernphysik, Universität zu Köln, D-50937 Köln, Germany \\ ${ }^{b}$ Heavy Ion Laboratory, Warsaw University, 02-093 Warsaw, Poland \\ ${ }^{c} K U$ Leuven, Instituut voor Kern- en Stralingsfysica, B-3001 Leuven, Belgium \\ ${ }^{d}$ Gesellschaft für Schwerionenforschung, 64291 Darmstadt, Germany \\ ${ }^{2}$ PH Department, CERN 1211, Geneva 23, Switzerland \\ ${ }^{f}$ CEA Saclay, Service de Physique Nucléaire, Gif-sur-Yvette, France \\ ${ }^{g}$ Oliver Lodge Laboratory, University of Liverpool, Liverpool, L69 7ZE, UK \\ ${ }^{h}$ Department of Physics and Astronomy, University of Edinburgh, Edinburgh, EH9 3JZ, UK \\ ${ }^{i}$ Physik Dept. E12, Technische Universität München, D-85748 Garching, Germany \\ ${ }^{j}$ Physics Department, Ludwig-Maximilian University, München, Germany \\ ${ }^{k}$ Department of Physics, University of York, Heslington, York, YO10 5DD, UK \\ 'Institut für Kernphysik, Technische Universität Darmstadt, 64289 Darmstadt, Germany \\ ${ }^{m}$ Institute for Nuclear Research and Nuclear Energy, 1784 Sofia, Bulgaria \\ ${ }^{n}$ Max-Planck Institute of Nuclear Physics, Heidelberg, Germany
}

\begin{abstract}
B}\left(\mathrm{E} 2 ; 2_{1}^{+} \rightarrow 0_{1}^{+}\right)$values have been measured for the unstable nuclei ${ }^{88} \mathrm{Kr}(\mathrm{N}=52)$ and ${ }^{92} \mathrm{Kr}(\mathrm{N}=56)$ using projectile Coulomb excitation at ISOLDE, CERN. With this experiment the local maximum in $\mathrm{E}\left(2_{1}^{+}\right)$in ${ }^{92} \mathrm{Kr}$ and the role of the $\mathrm{N}=56$ subshell closure can be studied.
\end{abstract}

Keywords: Coulomb excitation, collectivity, deformation PACS: 25.70.DE, 21.10.Ky

The $Z=36$ krypton isotopes are located in the middle of the $Z=28$ to $Z=50$ shell. In this region many interesting nuclear structure phenomena were observed. The $Z=38$ strontium nuclei, as well as the $\mathrm{Z}=40$ zirconium nuclei, show a sharp phase transition from spherical to very deformed nuclei towards $\mathrm{N}=60$ that is unique over the whole nuclear chart. It would be interesting to know if, for neutron-rich krypton isotopes, a similar phase transition appears, but $\mathrm{B}\left(\mathrm{E} 2 ; 2_{1}^{+} \rightarrow 0_{1}^{+}\right)$values were known up to the last stable isotope ${ }^{86} \mathrm{Kr}$ only.

Furthermore, there is a strong shell effect at $\mathrm{N}=56$ (filled $v\left(d_{5 / 2}\right)$ subshell) for zirconium isotopes, resulting in a sharp peak in $\mathrm{E}\left(2_{1}^{+}\right)$as both proton and neutron orbitals are

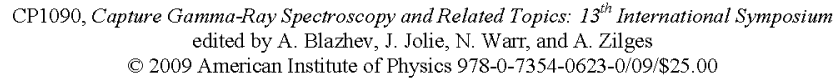


completely filled ("reinforced shell closure"). In the $A=100$ region no other nucleus shows a peak in $\mathrm{E}\left(2_{1}^{+}\right)$at $\mathrm{N}=56$, except the ${ }^{92} \mathrm{Kr}$ nucleus where the $2_{1}^{+}$state is located slightly higher with respect to the neighbouring even-A krypton nuclei. This local maximum is not as strong as in the case of the zirconium chain, but, as was pointed out by [1], "the effect in ${ }^{92} \mathrm{Kr}$ is remarkable when regarding the $\mathrm{E}\left(2_{1}^{+}\right)$trends in the isotones with $(Z=40 \pm 2)$ and $(Z=40 \pm 4)$ ".

We measured $B(E 2)$ values for the unstable ${ }^{88} \mathrm{Kr}$ and ${ }^{92} \mathrm{Kr}$ nuclei. The radioactive krypton beams from the REX-ISOLDE LINAC were guided onto thin layers of ${ }^{12} \mathrm{C}$ and ${ }^{109} \mathrm{Ag}$, respectively. The MINIBALL spectrometer, equipped with $8 \mathrm{HPGe}$ triple clusters was used to collect the gamma rays in coincidence with scattered particles, that were detected with a silicon strip detector. Using the Coulomb excitation analysis code GOSIA the $\mathrm{B}\left(\mathrm{E} 2 ; 2_{1}^{+} \rightarrow 0_{1}^{+}\right)$for both $\mathrm{Kr}$ nuclei could be determined relative to the

${ }^{109} \mathrm{Ag}$ target excitation. Our preliminary results are $\mathrm{B}\left(\mathrm{E} 2{ }^{88} \mathrm{Kr}, 2_{1}^{+} \rightarrow 0_{1}^{+}\right) \approx 8 \mathrm{~W} . \mathbf{u}$. and $\mathrm{B}\left(\mathrm{E} 2 ;{ }^{92} \mathrm{Kr}, 2_{1}^{+} \rightarrow 0_{1}^{+}\right) \approx 17$ W.u., both with errors of about $10-15 \%$.

Our observation of an increased $\mathrm{E} 2$ strength for ${ }^{92} \mathrm{Kr}$ is in contrast to what was supposed before. As the energies of the $2_{1}^{+}$states in ${ }^{88} \mathrm{Kr}$ and ${ }^{92} \mathrm{Kr}$ nuclei are almost equal, a similar E2 strength was expected referring to the Grodzins phenomenological relation [2]. Currently we do not have a simple explanation for our finding. Furthermore it is remarkable that $\mathrm{B}(\mathrm{E} 2)$ values of krypton isotopes do not behave like the neighbouring strontium isotopes, where $\mathrm{B}(\mathrm{E} 2)$ values are small and do not vary much until $\mathrm{N}=60$. Based on similarities in the level schemes of odd $\mathrm{Sr}$ and $\mathrm{Kr}$ nuclei, it was concluded in [3] that there is no signature of increased collectivity at low excitation energy in krypton nuclei, whereas our results show an enhanced quadrupole collectivity at $\mathrm{N}=56$ already. This rather leads to a conclusion of a smooth onset of deformation in neutron rich krypton isotopes. However, as the $\mathrm{B}(\mathrm{E} 2)$ value goes up, the $\mathrm{N}=56$ subshell closure seems not to have big influence at $Z=36$ and will most likely not explain the local maximum in $\mathrm{E}\left(2_{1}^{+}\right)$.

In [4], mean square charge radii $\delta\left\langle r^{2}\right\rangle$ of krypton nuclei were measured and compared to calculated values from the liquid drop model including static and dynamic deformation. Based on the Grodzins relation, neutron rich krypton nuclei were proposed to be soft against octupole deformation, a common phenomenon in the $A=100$ region. Octupole deformation may then explain the local maximum in $\mathrm{E}\left(2_{1}^{+}\right)$. However, after recalculating $\delta\left\langle r^{2}\right\rangle$ using our experimental E2 matrix elements we can explain the measured values without using the octupole degree of freedom. In the future, mean field calculations may bring insight into the question why $\mathrm{E}\left(2_{1}^{+}\right)$peaks at $\mathrm{N}=56$ and how collectivity evolves towards $\mathrm{N}=60$.

\section{REFERENCES}

1. K.-L. Kratz, H. Gabelmann, B. Pfeiffer, P. Möller, F. Nowacki, Z. Phys. A -Atomic Nuclei 330, 229-230 (1988).

2. L. Grodzins, Phys. Lett. 2, 88 (1962).

3. G. Lhersonneau, A. Wöhr, B. Pfeiffer, K.-L. Kratz, Phys. Rev. C. 63, 034316 (2001).

4. M. Keim, E. Arnold, W. Borchers, U. Georg, A. Klein, R. Neugart, L. Vermeeren, R.E. Silverans, P. Lievens, Nucl. Phys. A 586, 219-239 (1988). 